\title{
Influence of lincomycin-spectinomycin treatment on the outcome of Enterococcus cecorum infection and on the cecal microbiota in broilers
}

Jana Schreier ${ }^{1}$, Daniela Karasova ${ }^{2}$, Magdalena Crhanova ${ }^{2}$, Ivan Rychlik ${ }^{2}$, Silke Rautenschlein ${ }^{1}$ and Arne Jung ${ }^{1^{*}}$ (D)

\begin{abstract}
Background: Enterococcus cecorum (EC) is one of the main reasons for skeletal disease in meat type chickens. Intervention strategies are still rare and focus mainly on early antibiotic treatment of the disease, although there are no data available concerning the effectivity of this procedure. The present study aimed to investigate the effectivity of early lincomycin-spectinomycin treatment during the first week of life after EC-infection. Furthermore, the impact of lincomycin-spectinomycin treatment and EC infection on the development of cecal microbiota was investigated.
\end{abstract}

Methods: A total of 383 day-old broiler chicks were randomly assigned to four groups (non-infected and non-treated, non-infected and treated, EC-infected and non-treated, and EC-infected and treated). The EC-infected groups were inoculated orally with an EC suspension at the day of arrival and at study day 3 . The treatment groups were treated with lincomycin-spectinomycin via the drinking water for six consecutive days, starting two hours after the first inoculation. Necropsy of 20 chickens per group was performed at study days 7, 14, 21, and 42. Bacteriological examination via culture and real-time PCR was performed to detect EC in different extraintestinal organs. Cecal samples of nine chickens per group and necropsy day were analyzed to characterize the composition of the cecal microbiota.

Results: No clinical signs or pathologic lesions were found at necropsy, and EC was not detected in extraintestinal organs of the EC-infected and treated birds. Lincomycin-spectinomycin promoted the growth of the bacterial genus Escherichia/Shigella and reduced the amount of potentially beneficial Lactobacillus spp. in the ceca regardless of EC-infection. Unexpectedly, the highest abundances of the genus Enterococcus were found directly after ending antibiotic treatment in both treatment groups, suggesting the growth of resistant enterococcal species. EC was not detected among the most abundant members of the genus Enterococcus. Oral EC-infection at the first day of life did not influence the development of cecal microbiota in the present study.

Conclusions: Lincomycin-spectinomycin treatment during the first week of life can prevent the EC-associated disease in broiler type chickens and has a direct impact on the development of the cecal microbiota. The low abundance of EC in the ceca of infected chickens underlines the pathogenic nature of the disease-causing EC strains. Further research on alternative prevention and intervention strategies is needed with regard to current efforts on reducing the use of antibiotics in livestock animals.

*Correspondence: arne.jung@tiho-hannover.de

${ }^{1}$ Clinic for Poultry, University of Veterinary Medicine Hannover, Buenteweg 17, 30559 Hannover, Germany

Full list of author information is available at the end of the article

(c) The Author(s) 2022. Open Access This article is licensed under a Creative Commons Attribution 4.0 International License, which permits use, sharing, adaptation, distribution and reproduction in any medium or format, as long as you give appropriate credit to the original author(s) and the source, provide a link to the Creative Commons licence, and indicate if changes were made. The images or other third party material in this article are included in the article's Creative Commons licence, unless indicated otherwise in a credit line to the material. If material is not included in the article's Creative Commons licence and your intended use is not permitted by statutory regulation or exceeds the permitted use, you will need to obtain permission directly from the copyright holder. To view a copy of this licence, visit http://creativecommons.org/licenses/by/4.0/. The Creative Commons Public Domain Dedication waiver (http://creativeco mmons.org/publicdomain/zero/1.0/) applies to the data made available in this article, unless otherwise stated in a credit line to the data. 
Keywords: Enterococcus cecorum, Infection, Cecal microbiota, Lincomycin, Spectinomycin, Broilers

\section{Background}

The bacterial species Enterococcus cecorum (EC) has become a major cause of disease outbreaks in the broiler industry worldwide [1-8]. The EC-associated disease, also called "enterococcal spondylitis" or "kinky back", leads to an increase in mortality and therapy costs $[2,4$, 7]. The typical course of the disease is characterized by a septic phase during the first three weeks of the production cycle, followed by the skeletal phase, which lasts from week three until the end of the cycle [9]. During the septic phase, affected birds can be asymptomatic or show non-specific symptoms such as depression, ruffled feathers, and retarded growth. Pericarditis and hepatitis are often found at this stage [2]. The first signs of lameness mark the onset of the skeletal phase of the disease. Affected birds suffer from progressive lameness and ataxia. Completely paralyzed birds are often seen in a typical sitting position and should be culled, as they are not able to reach feed or water anymore [4]. Necropsy reveals the main reason for paralysis: necrotic abscess material at the free thoracic vertebra constricting the spinal canal [7]. Another reason for lameness is femoral head osteomyelitis, which can lead to complete destruction of the cartilage and the underlying bone [10].

The fecal-oral transmission route is most likely in EC pathogenesis [11]. EC is known to be a commensal in the chicken's intestine and was found in other birds and mammals as well [12]. Furthermore, it is thought to become the major enterococcal species in the gut of 12-week-old chickens [13]. Commensal strains start to colonize the gut in the third week of life. In contrast, pathogenic EC strains can be detected in ceca of chicks during the first week of life and may thus have a competitive advantage in colonizing the gut [9].

The role of the intestinal barrier function and the microbiota in enterococcal infections has not been the focus of studies on the EC infection until now [9]. Coinfection with other pathogens and immunosuppression have been thought to play a significant role in the EC pathogenesis $[11,14]$. Unfortunately, no clear predisposing infectious factors could be identified so far. Different virulence factors known from other enterococcal species were found in some pathogenic EC strains from clinical cases, but their role in EC pathogenesis remains unclear [15-17]. To date, it is still unknown how EC translocates from the intestine to other tissues [14].

Early detection of the pathogen and diagnosis of the associated disease are important, as therapy has to start early to avoid increasing costs over time, and therapy of skeletal lesions in affected animals is not successful [18]. According to the literature, the drug of choice in treating the EC-associated disease is Amoxicillin [7, 8]. Enterococci are known to have a high level of antimicrobial resistance [19] and this has to be considered when treating the EC-infection. EC has been shown to be multidrug resistant in several studies, and differences between commensal and pathogenic strains are usually found [16, 20-22]. Resistance to lincomycin was frequently detected in commensal isolates, whereas pathogenic isolates were often susceptible to this agent $[16,22]$. Resistance to spectinomycin is even less frequent in EC isolates from ceca and spine lesions [20].

In the European Union (EU), lincomycin and spectinomycin are available as a combined soluble powder and approved for use in the drinking water in chickens and swine. The indication for use in chickens is the treatment of chronic respiratory diseases caused by Mycoplasma gallisepticum and Escherichia coli, and associated with low mortality rates [23].

The chicken gut microbiota is known to play an important role in health and disease. Extensive research has been conducted and reviewed intensively in recent years. Several studies showed that the cecal microbiota of healthy broilers comprises mainly three different phyla: Firmicutes, Bacteroidetes, and Proteobacteria [24-26]. Different factors, such as diet, age, and housing are thought to have a direct impact on the development and constitution of intestinal microbiota [26-29]. The chicken intestinal microbiota is most complex in the cecum and is thought to intensively interact within the microbial population itself as well as with the host's immune system. The presence or absence of beneficial bacteria was linked directly to performance in meat type chickens [30]. As a result of high hygiene standards in modern broiler production, newly hatched chicks are relatively naïve regarding bacterial colonization of the intestine. Consequently, there is high variation in the composition of the cecal microbiota during the first two weeks of life. It is possible to manipulate the establishment of a stable bacterial community, and thus influence the health and productivity in the birds [31,32]. There is no data available on the impact of early cecal EC colonization on the development of the intestinal microbiota. Antibiotic treatment is known to reduce the stability of microbiota in the gut and increase antimicrobial resistance [25].

Based on field observations, lincomycin-spectinomycin is used to treat EC-infections in Germany. Although 
extensive research on the microbial community in the chicken's intestine has been conducted in recent years, there is no information on the effect of lincomycin-spectinomycin on the gut microbiota in broilers. Thus, the objective of this study was to investigate the impact of lincomycin-spectinomycin treatment on the EC infection and the gut microbiota in broilers.

\section{Results}

\section{Clinical signs and pathology}

In this experiment, symptoms of the EC-associated disease and typical gross lesions during the skeletal phase were only seen in the EC-infected, non-treated group (EN). The first non-specific symptoms were recorded at study day 13, and lameness was seen from study day 17 until the end of the study. In total, $36.9 \%$ of the birds in group EN showed non-specific symptoms including depression, ruffled feathers, and closed eyes, while $13.1 \%$ were found to be lame. Pericarditis was the most common gross lesion (26.2\%), whereas hepatitis was found in $13.1 \%$ of the chickens. At study days 21-42, birds were also checked for spondylitis and femoral head osteomyelitis during regular and irregular necropsies. Spondylitis (15.9\%) was found more often than femoral head osteomyelitis $(4.5 \%)$. The non-infected groups $(\mathrm{NN}, \mathrm{NL})$ and the EC-infected group treated with lincomycin-spectinomycin (EL) did not show any EC-associated clinical signs or gross lesions throughout the trial. In group EN, four of 84 birds were euthanized due to animal welfare reasons. Necropsy was performed on all dead and euthanized birds according to the regular necropsy protocol. Three of these birds were EC-positive on culture, and spondylitis was found at necropsy. In group NN, two broilers were found dead in the first week (early chick mortality without any lesions), and in group NL, one broiler was euthanized due to ascites syndrome.

\section{Qualitative microbiology via culture and real-time PCR}

In total, $28.57 \%$ of the birds in the EN group were ECpositive on culture (Fig. $1 \mathrm{~A}$ ). EC was mainly isolated from the spleen $(21.43 \%)$, followed by the heart and liver (9.52\% each). Isolation rates from these organs and the overall isolation rate were significantly higher compared to the other groups $(p \leq 0.05)$, as EC was not recovered from the birds in any other group. The number of ECpositive chickens in the EN group peaked at study day 21, with nine of 20 birds (45\%) being EC-positive in either one or more of the examined organs (heart, liver, and spleen). The free thoracic vertebra (FTV) and the femoral heads $(\mathrm{FH})$ were only bacteriologically examined when gross lesions were detected. Throughout the whole trial, $8.33 \%$ of the broilers in the EN group developed EC-positive gross lesions at the FTV. A smaller amount of birds (3.57\%) had osteomyelitis lesions at the femoral heads that were positive for EC.

In real-time PCR, EC was detected in the spleen, the FTV, and the FHs of the EN group throughout the trial (Fig. 1 A). On comparing the results from the different organs at the time points where all the respective organs were sampled (study days 21 and 42), EC was detected mainly in the FTV (30\%), followed by the spleen $(22 \%)$, and the femoral heads (18\%). In total, 50\% (22/44) of the birds were EC-positive in either one or more of the examined organs. Spleen samples from study days 7 and 14 were additionally analyzed. The number of EC-positive spleens increased during the first three weeks and peaked at study day 21 (Fig. 1B). The Kappa coefficient and McNemar's test were calculated for comparison of the detection rates of EC in the spleen via culture and
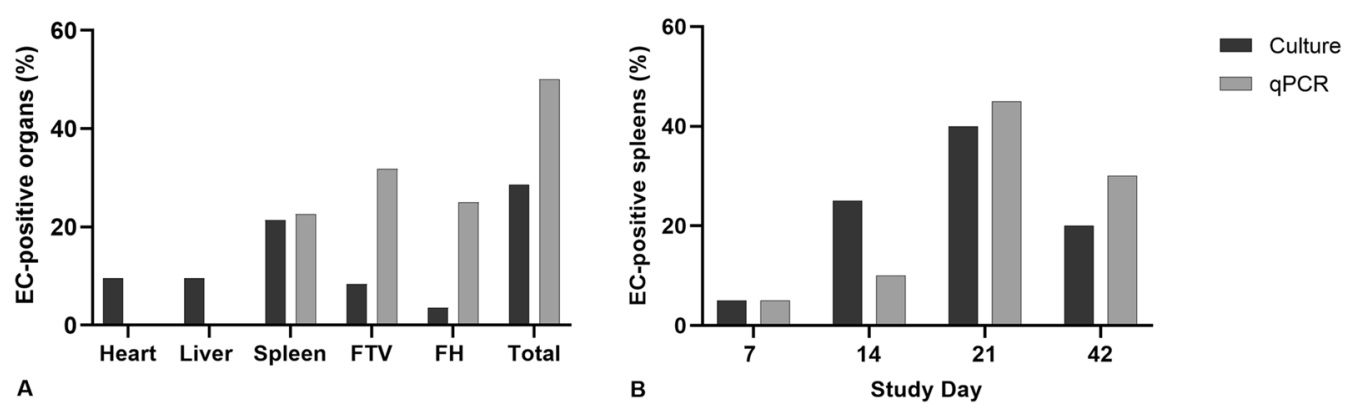

Fig. 1 Microbiological examination via culture and real-time PCR in the EC-infected, non-treated group (EN). A EC-positive organs detected via culture and real-time PCR. All the hearts, livers and spleens were examined for EC via culture. FTV and FH were only examined on culture when gross lesions were detected at necropsy. Real-time PCR was performed for all the spleens at all necropsy days, and the free thoracic vertebra (FTV) and femoral heads (FH) at study days 21 and 42. Ct values below 36 were considered positive. B EC-positive spleens detected via culture and real-time PCR per study day. No significant differences in EC-detection were found between the two methods ( $p=1.0$, McNemar's test) and results were substantially concordant $(\mathrm{K}=0.6416)$ 
real-time PCR. Results from culture and real-time PCR were substantially concordant $(\mathrm{K}=0.6416)$ and no significant difference between the two methods was found ( $p$ $=1.000)$. The number of EC-positive FTVs and FHs was highest at the end of the study. At study day $42,45 \%$ of the FTVs and 25\% of the FHs were EC-positive.

\section{EC detection in the cecum via quantitative real-time PCR}

During the first three weeks, almost $100 \%$ of the tested broilers in the EC-infected, non-treated group (EN) were EC-positive in the cecum (Fig. 2). At study day 7, immediately after the end of antibiotic treatment, EC-DNA was detected in $20 \%$ of the birds in the EC-infected group with antibiotic treatment (EL). In this group, EC was not detected in the following two weeks (study days 14 and 21). However, at study day 42 , EC was detected in all the birds from group EL. Furthermore, EC was found in approximately $45 \%$ of the birds in groups NL and EN at study day 42 . The non-infected, non-treated group $\mathrm{NN}$ remained EC-negative throughout the study.

\section{Characterization of cecal microbiota Sequencing coverage and depth}

A total number of 154 cecal samples were analyzed. We obtained 7,214,356 reads, which led to an average coverage of 46,846 reads. The lowest coverage was 10,370 reads, the highest coverage 140,978 reads. These reads were distributed among 41,315 operational taxonomic units (OTUs).

\section{Phylum level}

In total, 22 different phyla were identified in this experiment. Independent of study day or group, the majority of these phyla (>99\%) was formed by Firmicutes (76.87\%), Proteobacteria (12.45\%), and Bacteroidetes (9.71\%). The relative abundance of these phyla changed over time. On the first day of life, Firmicutes formed the majority of phyla identified in the ceca $(98.56 \%)$, followed by Proteobacteria (0.75\%), and Bacteroidetes (0.4\%). At the first necropsy day, which was study day 7 , and directly after the end of treatment with lincomycin-spectinomycin, clear differences between the treated and untreated groups were found. In groups NN and EN, Firmicutes represented the majority of phyla (84.95 and $90.17 \%$, respectively), followed by Proteobacteria (15.02 and 9.79\%, respectively). However, in groups NL and EL, Proteobacteria represented the majority of phyla (55.56 and $50.29 \%$, respectively), followed by Firmicutes (44.4 and $49.66 \%$, respectively). In the following weeks of the experiment, these differences became less clear and the relative abundance of Firmicutes increased in both groups treated with lincomycin-spectinomycin until study day 21 (>90\%). At study days 14 and 21, Firmicutes represented the predominant phylum in all four groups (> 70\%). At study day 42, Firmicutes was still the phylum with the highest abundance in all four groups $(>50 \%)$, followed by Bacteroidetes (the relative abundance ranged between $20.53 \%$ (EN) and $42.45 \%(\mathrm{NN})$ ) (Fig. 3).

\section{Family and genus level}

To provide deeper insight into the microbial composition in the ceca of the different groups and the development over time, we performed further analysis at family and genus level. Data are shown in Fig. 4A (family level) and Fig. 4B (genus level). In total, 426 different genera from 173 bacterial families were identified in this experiment.

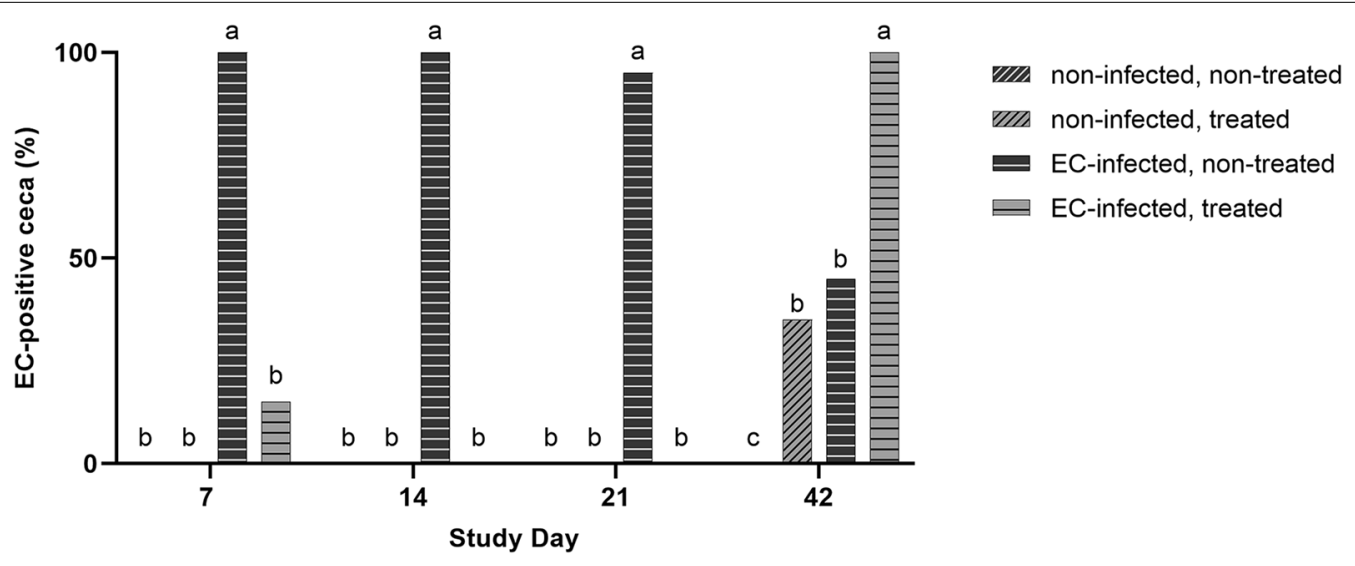

Fig. 2 Cecal colonization by EC. Birds were infected with EC at study day 1, and treated groups received lincomycin-spectinomycin via the drinking water from study day 1 to 6 . Samples were analyzed via real-time PCR and Ct values below 36 were considered positive. Different letters indicate significant differences between the groups per study day $(p \leq 0.05)$. Comparison between the groups was made for each study day by using Fisher's exact test. $p$-value adjustments for multiple testing were performed by using the Bonferroni-Holm correction method. $N=20$ per group and study day 


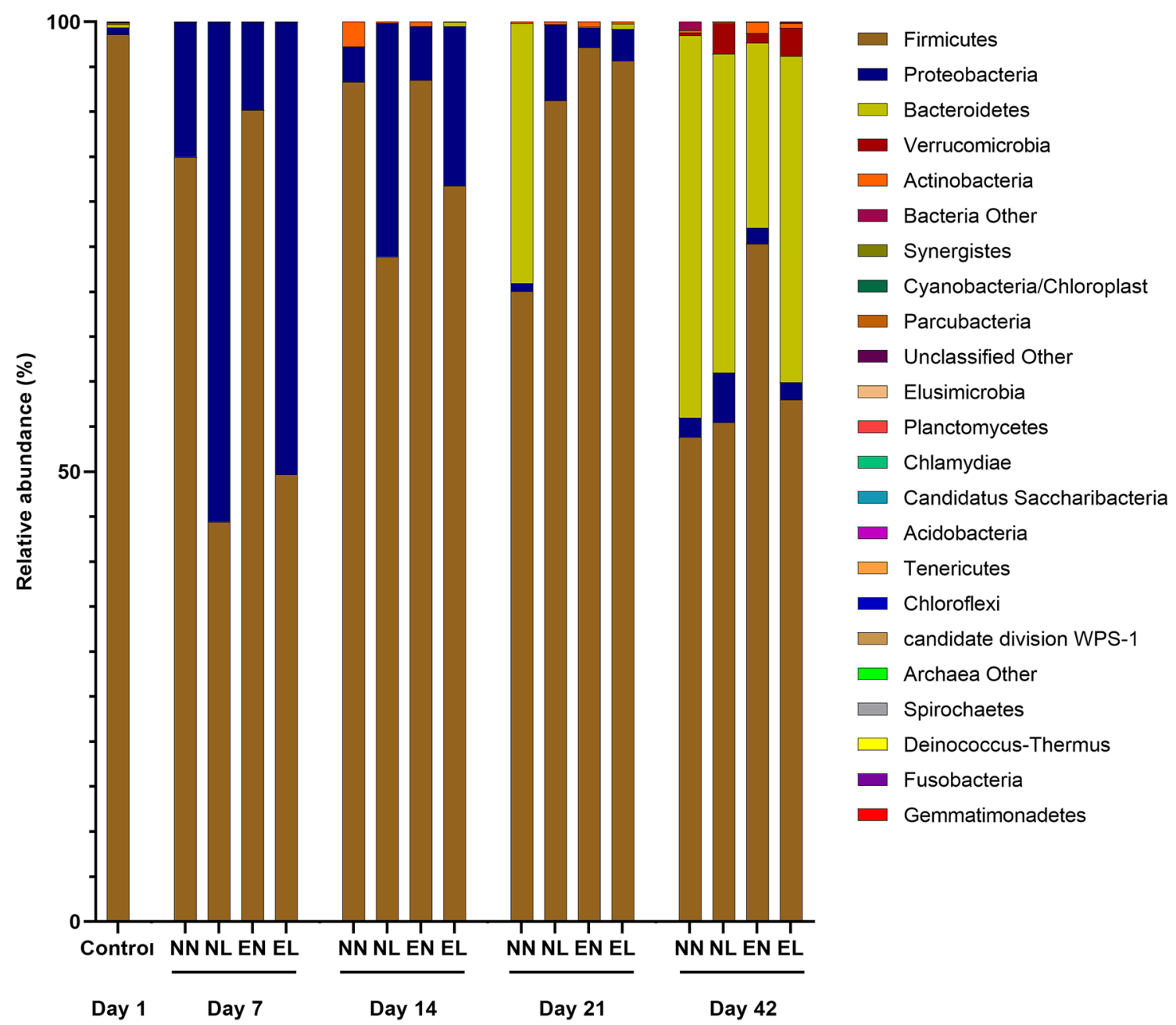

Fig. 3 Characterization of cecal microbiota at phylum level. Data were analyzed using Qiime. Relative abundances (\%) of each phylum are presented per study day and group. $N=9$ per group and study day; $N N$ non-infected, non-treated, $N L$ non-infected, treated with lincomycin-spectinomycin, EN EC-infected, non-treated, EL EC-infected, treated with lincomycin-spectinomycin

At genus level, control birds at study day 1 (EC-negative status) were mainly colonized by the genus Clostridium sensu stricto (95\%, family Clostridiaceae 1), followed by Clostridium XIVa (0.6\%, family Lachnospiraceae, phylum Firmicutes) and Escherichia/Shigella (0.56\%, family Enterobacteriaceae, phylum Proteobacteria). After the first week of life and right after the end of treatment at study day 7, the microbial composition had become more diverse in all four groups. In comparison to the first day of life, Clostridium sensu stricto was far less abundant in all four groups compared to the first study day, especially in the two treatment groups $(6.0 \%$ in $\mathrm{NN}, 0.26 \%$ in $\mathrm{NL}$, $7.59 \%$ in EN, and $0.16 \%$ in EL, respectively). In groups NN and EN, Clostridium XIVa (14.03\% and 16.19\%, respectively) and Lactobacillus (family Lactobacillaceae, phylum Firmicutes; $10.93 \%$ and $13.56 \%$, respectively) were some of the most abundant genera. Both of these genera were far less abundant in the two treatment groups NL and EL $(<1 \%)$. In groups NL and EL, the most abundant genus was Escherichia/Shigella (> 50\%), followed by Blautia (> 20\%, family Lachnospiraceae). The genus Escherichia/Shigella was also found in the untreated groups $\mathrm{NN}$ and EN at study day 7, but at a relatively lower abundance (14.98\% and $9.77 \%$, respectively). One week later, at study day 14, the relative abundance of Escherichia/Shigella had decreased in groups NL and EL (25.79\% and $17.68 \%$, respectively). A further decrease towards the end of the study was seen as the microbial composition became more complex and various other genera were found in all four groups. In contrast to the first three necropsy days, the abundance of different 


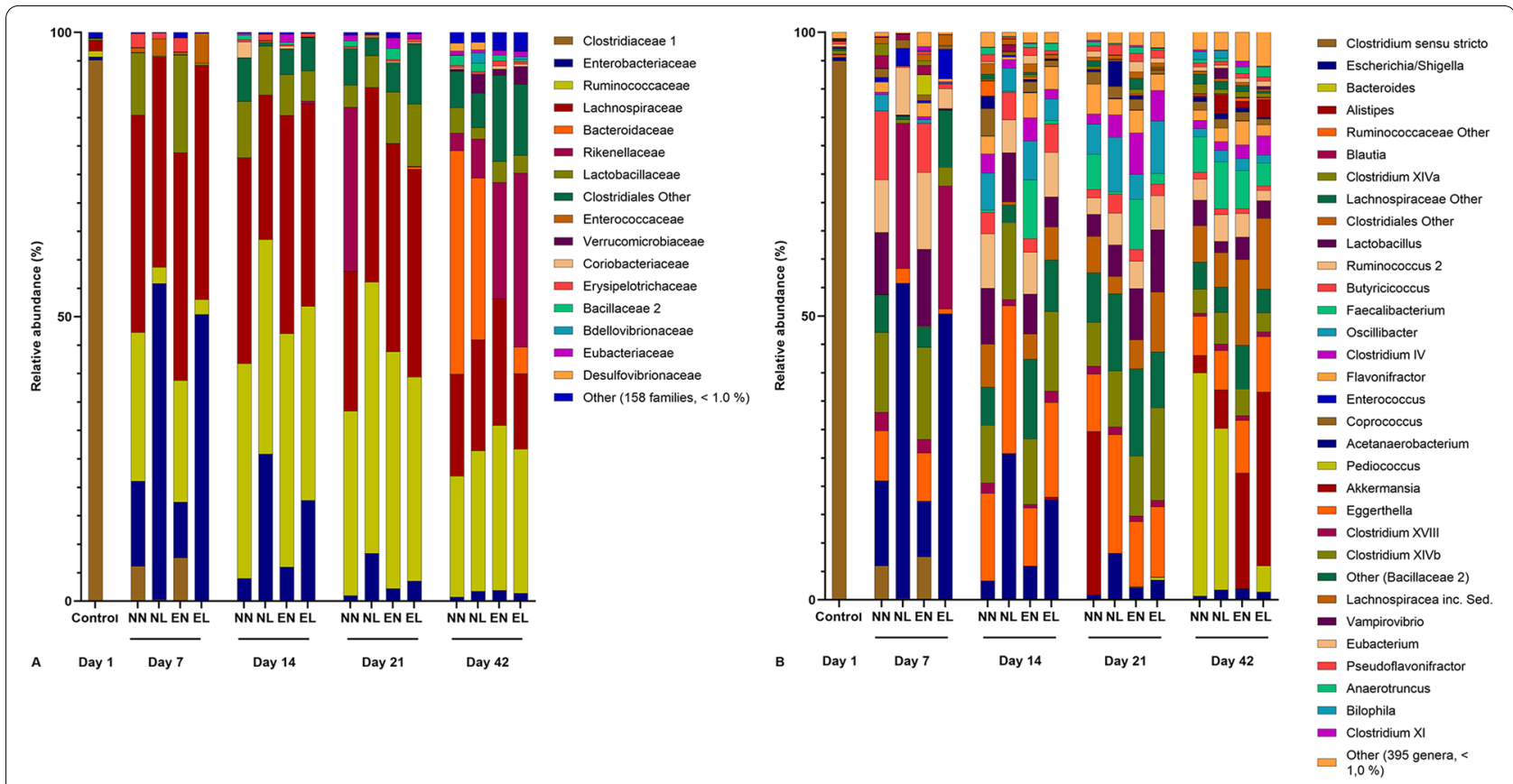

Fig. 4 Characterization of cecal microbiota at family level A and genus level (B). Data were analyzed using Qiime. Relative abundances (\%) of each family or genus are presented per study day and group. A total of 158 families and 395 genera were summarized as "Other" because the overall average of the relative abundance was below $1.0 \% . N=9$ per group and study day, NN non-infected, non-treated, $N L$ non-infected, treated with lincomycin-spectinomycin; EN EC-infected, non-treated, EL EC-infected, treated with lincomycin-spectinomycin

genera in groups NN and NL was highly similar to each other as was the case in groups EN and EL at study day 42.

Alpha diversity indices and principal coordinate analysis for visualization of beta diversity further underlined differences seen at family and genus level. As expected, at the end of antibiotic treatment at study day 7 , the untreated groups (NN, EN) were colonized by significantly more species than the lincomycin-spectinomycin treated groups (NL, EL; Table 1). Similar significant differences were found at that day for the Chaol index, which estimates species richness, and the Shannon index,

Table 1 Alpha diversity in cecal samples

\begin{tabular}{|c|c|c|c|c|c|c|c|c|c|}
\hline \multirow{2}{*}{$\begin{array}{l}\text { Study day } \\
\text { Group }\end{array}$} & \multirow{2}{*}{$\begin{array}{l}1 \\
\text { Control }\end{array}$} & \multicolumn{4}{|l|}{7} & \multicolumn{4}{|l|}{14} \\
\hline & & NN & NL & EN & EL & NN & $\mathrm{NL}$ & EN & EL \\
\hline $\begin{array}{l}\text { Observed } \\
\text { species }\end{array}$ & $256 \pm 74$ & $606 \pm 159^{a}$ & $315 \pm 54^{b}$ & $732 \pm 209^{a}$ & $351 \pm 60^{b}$ & $1917 \pm 462^{\mathrm{a}}$ & $1262 \pm 351^{b}$ & $3161 \pm 919^{a}$ & $2264 \pm 751^{a}$ \\
\hline $\begin{array}{l}\text { Chao1 esti- } \\
\text { mate }\end{array}$ & $515 \pm 114$ & $1118 \pm 296^{a}$ & $613 \pm 92^{b}$ & $1425 \pm 356^{a}$ & $732 \pm 137^{b}$ & $4088 \pm 978^{a c}$ & $2984 \pm 921^{a}$ & $7427 \pm 1948^{b}$ & $5356 \pm 1642^{b c}$ \\
\hline Shannon index & $0.59 \pm 0.27$ & $4.23 \pm 0.38^{a}$ & $1.85 \pm 0.17^{b}$ & $4.39 \pm 0.45^{a}$ & $2.15 \pm 0.68^{b}$ & $5.72 \pm 0.19^{a}$ & $4.15 \pm 0.39^{b}$ & $6.49 \pm 0.44^{c}$ & $5.51 \pm 0.61^{a}$ \\
\hline \multicolumn{2}{|l|}{ Study day } & \multicolumn{4}{|l|}{21} & \multicolumn{4}{|l|}{42} \\
\hline Group & & NN & NL & EN & EL & NN & $\mathrm{NL}$ & EN & EL \\
\hline $\begin{array}{l}\text { Observed } \\
\text { species }\end{array}$ & & $2630 \pm 880^{a}$ & $2075 \pm 396^{a}$ & $2279 \pm 836^{a}$ & $1358 \pm 388^{b}$ & $1382 \pm 275^{\mathrm{a}}$ & $1458 \pm 416^{a}$ & $2067 \pm 568^{a}$ & $1801 \pm 777^{a}$ \\
\hline $\begin{array}{l}\text { Chao1 esti- } \\
\text { mate }\end{array}$ & & $6923 \pm 1831^{a}$ & $4524 \pm 893^{b c}$ & $5438 \pm 1609^{a c}$ & $3602 \pm 800^{b}$ & $3971 \pm 796^{a}$ & $3890 \pm 1097^{a b}$ & $5867 \pm 1308^{b}$ & $4840 \pm 1810^{a b}$ \\
\hline Shannon index & & $5.77 \pm 0.53^{a}$ & $5.84 \pm 0.24^{a}$ & $6.35 \pm 0.27^{b}$ & $6.10 \pm 0.24^{a b}$ & $5.15 \pm 0.36^{a}$ & $5.76 \pm 0.33^{b}$ & $6.38 \pm 0.31^{c}$ & $5.60 \pm 0.73^{a b}$ \\
\hline
\end{tabular}

Different superscript letters indicate significant differences between the groups per study day ( $p \leq 0.05$ ). Comparison between the groups was made by using the Kruskal-Wallis-Test and Mann-Whitney-U-Test, followed by the Bonferroni-Holm correction method for $p$-value adjustment for multiple testing. NN non-infected, nontreated, NL non-infected, treated with lincomycin-spectinomycin, EN EC-infected, non-treated, EL EC-infected, treated with lincomycin-spectinomycin 
which estimates species diversity and takes into account species richness and evenness. At study days 14, 21, and 42 , significant differences were found between groups for all three indices, but they were not the same for all three estimators.

Composition of the gut microbiota in nine chickens per group and day was visualized by principal coordinate analysis (PCoA) based on weighted and unweighted UniFrac distance metric (Additional file 1). In general, chickens belonging to the same group shared highly similar microbiota at one time point and clustered closely together. The microbial cluster from one group at a specific time point was distinct from other groups at the same time point or the same group at another time point. At study day 7, the two treatment groups clustered close to each other but were distinct from the untreated groups which formed two other closely related clusters. In contrast, the two EC-infected groups (EN, EL) clustered separately from the non-infected groups (NN, NL) at study day 42. Furthermore, the samples from the two uninfected groups were relatively more distinct from each other within their respective cluster.

\section{Genus Enterococcus}

Enterococcus is a member of the family Enterococcaceae within the phylum Firmicutes. Among the 2300 most abundant OTUs in our study, we could find nine sequences assigned to the genus Enterococcus. Three of them were identified as E. gallinarum, two as E. faecalis, one as E. faecium, and three could not be defined exactly but were most likely to be E. faecium, E. hirae or E. durans. None of these sequences belonged to Enterococcus cecorum. A closer look at the relative abundance of the genus Enterococcus in the four groups at all the given time points revealed an unexpected outcome. In control birds at the first study day as well as in all groups at study days 14, 21, and 42, Enterococcus was lowly abundant in the cecal microbiota $(<0.05 \%)$. However, at study day 7, Enterococcus was the fourth most abundant genus in groups NL and EL (3.03 and 5.21\%, respectively), but less abundant in groups $\mathrm{NN}$ and $\mathrm{EN}$ (0.83 and $0.42 \%$, respectively; Fig. 5).

\section{Discussion}

EC infections are one of the main problems in broiler production today. However, there is no data available concerning treatment of the disease. The aim of this study was to investigate an intervention strategy against ECassociated disease outbreaks. We examined the course of the EC-infection after inoculation on the first and third day of life, accompanied by an early onset of treatment with lincomycin-spectinomycin. In this experiment, the recommended dosage of lincomycin-spectinomycin ( $16.65 \mathrm{mg}$ lincomycin and $33.35 \mathrm{mg} / \mathrm{kg} \mathrm{BW}$ ) for chickens was used in the drinking water for six consecutive days after the first inoculation. The strain EC14/086/4/A was sensitive to lincomycin and spectinomycin in antimicrobial susceptibility testing using disk diffusion tests. The antibiotic treatment successfully prevented the onset of the disease. None of the typical EC-associated clinical symptoms or gross lesions were found in the EC-infected, lincomycin-spectinomycin treated birds (EL). Furthermore, in this group, EC was not detected via culture or real-time PCR in any of the extraintestinal examined organs throughout the experiment. In contrast, clinical symptoms and pathologic lesions of the EC-associated disease were seen in the EC-infected, non-treated group (EN). The detection rates via culture and real-time PCR

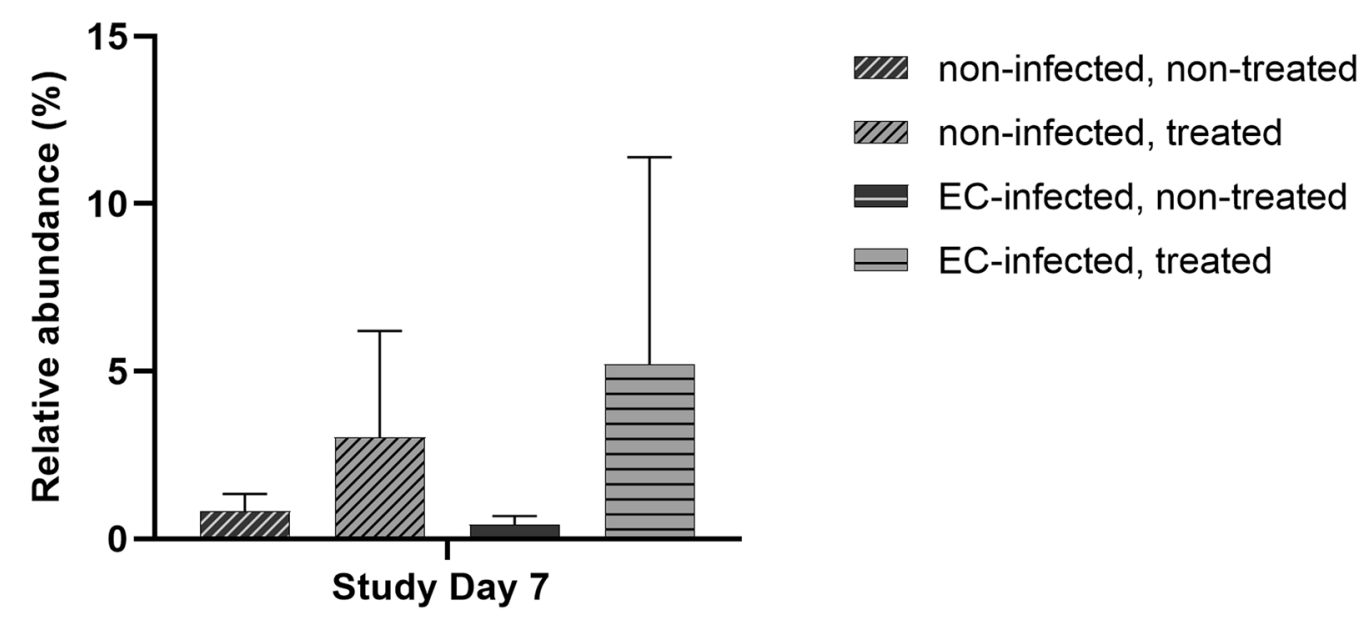

Fig. 5 Relative abundances of the genus Enterococcus at study day 7 in the four different study groups. Bars represent the mean and standard deviation. Treated treated with lincomycin-spectinomycin 
in this group correspond to morbidity rates reported from field outbreaks and experimental infection [2, 9, 14]. EC was frequently detected in the different examined extraintestinal organs and the typical course of the disease, including the septic and the skeletal phase, was successfully reproduced in this experimental group.

All the birds in group EN were colonized by EC throughout the first three weeks of the experiment. In contrast, EC-DNA was detected in $15 \%$ of the ceca in the EL group at study day 7, but not detected at all at study days 14 and 21, suggesting that no viable EC was left in this group after treatment. Interestingly, some of the birds of the respective group were colonized again at study day 42 . We cannot exclude that the birds in group EL were colonized again by the experimental strain EC14/086/4/A, since it could have survived in the environment of the birds. On the other hand, it is possible that another unknown and probably commensal EC strain colonized the birds. This question remains unanswered, as we were not able to isolate the strain and thus could not characterize it further. While the non-infected, untreated group $(\mathrm{NN})$ was not colonized by $\mathrm{EC}$ until the end of the study, $40 \%$ of the birds in the non-infected group treated with lincomycin-spectinomycin (NL) were colonized by EC at study day 42 . It can be hypothesized that this group was colonized by a commensal EC strain, but the origin of this strain remains unclear. Enterococci are known to be widespread in the environment of broilers and can be found in litter and feed [33]. Recently, it has been shown that EC is highly durable and can survive on different materials and under various environmental conditions for quite a long time [34]. Even though our experiment was conducted under strict hygiene conditions, it is still possible that EC entered the stable via the feed, the litter or other biotic or abiotic resources [35], as any other gut microbiota member detected in the ceca of all the chickens used in this study did.

Interestingly, there were considerably low abundances of the genus Enterococcus among the cecal microbiota of all the study groups at all investigated time points. At study day 7, the relative abundance of the genus Enterococcus was higher in the two groups treated with lincomycin-spectinomycin compared to the untreated groups. Resistance against lincomycin-spectinomycin of different enterococcal species from poultry was investigated in 2016 [36]. It was shown that the majority of all enterococcal isolates were resistant against lincomycin-spectinomycin. We therefore suggest that the antibiotic treatment suppressed the growth of major microbiota members but promoted the growth of other lincomycin-spectinomycin resistant Enterococcus species. Additionally, EC has been shown to be one of the most predominant enterococcal species in poultry, but less frequently isolated than $E$. faecalis and E. faecium [36]. This might explain the fact that we were not able to find sequences of EC among the top 2300 OTUs in the present study. In general, interaction within the cecal microbiota as well as between the microbes and the host is highly complex. Furthermore, specific bacteria associated with disease in chickens and humans (e.g. Clostridium difficile [37], Campylobacter spp. [29], and Salmonella enterica serovar Enteritidis [38]), have been studied in regard to their influence on the microbiota composition with divergent outcomes. Results from these studies led to the conclusion that not necessarily a single species but rather a combination of different bacterial groups and their complex interactions are of vital importance for the development of the cecal microbiota [37]. The low abundance of the genus Enterococcus in our study suggest that it is not necessary for EC to be a predominant species in the cecum to be able to cause the disease. This seems to be an important finding and underlines the pathogenic nature of disease-causing EC strains. Similar data have been found for Escherichia coli (E.coli), which is less abundant in the ceca of healthy chickens $[39,40]$. Nevertheless, pathogenic strains of $E$. coli are the causing agent of colibacillosis, the most common infectious bacterial disease in poultry [41].

EC-infection did not influence the development of the cecal microbiota and the species diversity in our study, as the composition of the cecal microbiota as well as alpha and beta diversity in the two untreated groups NN and EN was highly similar within the first two weeks of the experiment. Differences at later time points cannot be explained only based on the two influencing factors antibiotic treatment and EC-infection, but rather by several environmental factors, including litter and feed. It is most likely that the cecal microbiota within each physically separated experimental group developed individually until the end of the study. The development of cecal microbiota in chickens has been studied intensively in the past. Initial exposure of naïve chicks to different bacterial communities can lead to distinct microbiota [32], and differences in microbiota diversity depend on many factors such as age [37], diet [30], and litter [27]. Thus, finding possible intervention strategies to prevent bacterial diseases, such as prebiotics, probiotics or competitive exclusion, is very challenging [42]. Knowledge about the composition and development of cecal microbiota in young broiler chickens may be important for the development of preventive methods against the EC infection.

In addition to data on cecal colonization under ECinfection, the present study provides an overview on the development of cecal microbiota under lincomycinspectinomycin treatment. Usage of antimicrobials is known to disturb the homoeostasis of the gut microbiota [25]. Accordingly, we wanted to further characterize 
the effect of treatment on the development of the cecal microbiota throughout the experiment. We could show that antibiotic treatment has a direct impact on the composition of the cecal microbiota. Development of the cecal microbiota in untreated groups resembled the development of cecal microbiota in healthy broilers with Firmicutes, Proteobacteria, and Bacteroidetes, being the most abundant phyla in all experimental groups at all given time points $[24,25]$. The treatment groups NL and EL were colonized by members of the genus Escherichia/Shigella in high abundance after treatment. This leads to the suggestion that treatment with lincomycin-spectinomycin could enable colonization by other facultative pathogens such as multidrug-resistant avian pathogenic E. coli. Lincomycin is not active against $E$. coli [43]. Resistance against spectinomycin is widespread among avian E. coli isolates and often associated with multi-drug resistance $[44,45]$. Nevertheless, lincomycin-spectinomycin is approved for treatment of $E$. coli infections in poultry. However, antibiotic treatment of enterococcal infections with lincomycin-spectinomycin may lead to the selection of pathogenic E. coli and should be administered cautiously. Additionally, members of the genus Lactobacillus were frequently found in the untreated groups NN and EN, but far less so in the lincomycinspectinomycin treated groups NL and EL. Lactobacilli are described as beneficial bacteria in the literature and often used in pro- and synbiotics [46]. Several studies have shown that Lactobacillus spp. can decrease colonization of different bacteria, including Campylobacter jejuni [47], Salmonella enterica [48], and E. coli [49]. This leads to the suggestion that treatment with lincomycin-spectinomycin could not only enable pathogenic bacteria to excessively colonize the gut. Treatment also seems to reduce the amount of beneficial bacteria in the gut which could otherwise control potentially harmful bacteria.

Our results show that the early onset of treatment with lincomycin-spectinomycin can prevent the ECassociated disease in broiler chickens. In general, antibiotic resistance to lincomycin is relatively high, whereas resistance to spectinomycin and other aminoglycosides tends to be rather low in EC [50]. However, the antibiotic resistance profile of the respective pathogenic EC strain has to be taken into account when treating field outbreaks. The success of the treatment should be verified continuously in order to be able to react in time if treatment fails, as chronic bone lesion treatment in affected birds is impossible $[7,8]$. In regard to future perspectives and current efforts on reducing the use of antibiotics in livestock animals, other preventive methods are needed, which include identifying transmission routes, effective cleaning and disinfection, vaccination strategies, and potential use of probiotics. Further research is required to find suitable preventive intervention strategies.

\section{Conclusions}

In conclusion, treatment of the EC infection with lincomycin-spectinomycin successfully prevented the onset of the EC-associated disease in our study. In addition, this study provides first insights into the development of cecal microbiota during EC infection. Whereas antibiotic treatment had a direct impact on the composition of the cecal microbiota, surprisingly, no clear differences were found between the EC-infected and non-infected groups. Pathogenic EC strains seem to play a minor role within the microbial community in the ceca of broiler chickens, but nevertheless lead to severe disease outbreaks. Further research is needed to understand the role of the intestinal microbiota in EC pathogenesis and to find alternative preventive intervention strategies.

\section{Materials and methods}

\section{Animals and housing}

A total of 383 one-day-old broiler chicks (Ross 308, obtained from Brüterei Weser-Ems GmbH \& Co. KG, Visbek, Germany) were housed in floor pens on wood shavings in isolation units at the Clinic for Poultry, University of Veterinary Medicine Hannover, Foundation, Hannover, Germany. Upon arrival, 10 birds were submitted to necropsy and the remaining chicks were randomly divided into four groups. A broiler standard diet (Deuka, Deutsche Tiernahrung Cremer GmbH \& Co. KG, Duesseldorf, Germany) was fed ad libitum throughout the trial. Starter feed was provided from days 1 to 10 , followed by grower diet from days 11 to 35 , and finisher diet from day 36 to 42 (Table 2). The light program was $24 \mathrm{~h}$

Table 2 Composition of starter, grower, and finisher diet

\begin{tabular}{lccc}
\hline Ingredients & $\begin{array}{l}\text { Starter diet } \\
\text { (Days 1-10) }\end{array}$ & $\begin{array}{l}\text { Grower diet } \\
\text { (Days 11-35) }\end{array}$ & $\begin{array}{l}\text { Finisher diet } \\
\text { (Days 36-42) }\end{array}$ \\
\hline Crude protein (\%) & 21.50 & 20.00 & 18.00 \\
Crude fat (\%) & 4.90 & 5.00 & 2.80 \\
Crude cellulose (\%) & 3.90 & 3.30 & 3.00 \\
Crude ash (\%) & 5.40 & 4.90 & 5.40 \\
Lysine (\%) & 1.25 & 1.05 & 0.80 \\
Methionine (\%) & 0.55 & 0.50 & 0.40 \\
Calcium (\%) & 0.90 & 0.80 & 1.00 \\
Phosphorus (\%) & 0.60 & 0.50 & 0.60 \\
Sodium (\%) & 0.14 & 0.14 & 0.15 \\
Metabolizable & 12.40 & 12.40 & 12.00 \\
energy (MJ ME/kg) & & &
\end{tabular}


of light on the day of placement, followed by $15 \mathrm{~h}$ of light from 07:30 to 22:30 until the end of the study, and standard temperature conditions were adjusted throughout the experiment [51].

Data are summarized based on the manufacturer's indications (Deuka, Deutsche Tiernahrung Cremer GmbH \& Co. KG, Duesseldorf, Germany).

\section{Experimental set-up}

On the day of arrival, 10 birds were euthanized and checked for their EC-negative status (yolk sac, cecum). The remaining 373 birds were randomly divided into four groups and inoculation was performed. The first group was a non-infected, non-treated negative control group (NN). The second group was a non-infected group treated with Lincospectin ${ }^{\circledR}(222 \mathrm{mg} / \mathrm{g}$ lincomycin and $444.7 \mathrm{mg} / \mathrm{g}$ spectinomycin, Zoetis Deutschland $\mathrm{GmbH}$, Berlin, Germany) via the drinking water (NL). Birds in the third group were infected with EC14 but not treated with the antibiotics (EN). The fourth group was infected with EC14 and treated with Lincospectin ${ }^{\circledR}$ (EL). Birds were inoculated orally with $0.5 \mathrm{~mL}$ of an EC suspension at study day 1 directly after arrival, and again at study day 3. Unintentionally, for the first inoculation, the bacterial concentration in the inoculum was too low $\left(10^{4}\right.$ colony forming units (CFU) in $0.5 \mathrm{~mL}$ ), so a second inoculation was performed at study day 3 . On that day, birds were infected orally with $0.5 \mathrm{~mL}$ of an EC suspension containing $10^{8} \mathrm{CFU}$. After inoculation, chicks had no access to water for $2 \mathrm{~h}$. Birds in the treatment groups were treated with Lincospectin ${ }^{\circledR}$ via the drinking water for 4 days after the second inoculation until study day 6 . The dosage was $2.5 \mathrm{~g}$ Lincospectin ${ }^{\circledR}$ powder per $10 \mathrm{~L}$ water $(=75 \mathrm{mg} /$ $\mathrm{kg}$ body weight as recommended by the manufacturer). A daily monitoring of clinical signs, including depression, ruffled feathers, closed eyes (non-specific symptoms), and lameness, was performed in all four groups throughout the trial. Severely affected birds were euthanized and submitted to necropsy. Regular necropsies of 20 broilers per group were performed at study days 7, 14, 21 , and 42 . Gross lesions were documented at necropsy. The heart, liver, and spleen of all the birds were sampled for microbiological examination using Amies medium swabs (Hain Lifesciences GmbH, Nehren, Germany). Furthermore, dry swabs (Applimed SA, Châtel-St-Denis, Switzerland) from the spleen and the cecum were taken from all the birds for real-time PCR, and cecal samples were taken from nine chickens per group for the analysis of cecal microbiota. At study days 21 and 42, the free thoracic vertebra and the femoral heads were examined for pathologic lesions. Briefly, the respective sampling sites were exposed and cut sagittally in order to assess gross lesions at the cartilage and the underlying bone. Subsequently, dry swabs were taken from the bone marrow for real-time PCR and stored at $-20^{\circ} \mathrm{C}$ for at maximum six weeks until further analysis.

\section{Challenge isolates and preparation of the inoculate}

The EC isolate EC 14/086/4/A was used for challenge. This pathogenic strain was isolated from an EC-associated disease outbreak in a commercial broiler flock in 2014 and further characterized in a previous study [17]. The bacterial strain was thawed and grown on Columbia sheep blood agar (Oxoid GmbH, Wesel, Germany) at $37^{\circ} \mathrm{C}$ for $20 \mathrm{~h}$ under microaerophilic conditions. Subcultures were prepared on the following day and incubated for another $20 \mathrm{~h}$ before preparing the inoculum. Colony material was dissolved in physiological saline solution at room temperature up to an optical density of $1.1 \mathrm{McFar}-$ land (McF; DENSIMAT; BioMérieux, Nuertingen, Germany), which corresponds to an EC concentration of 2 * $10^{8}$ colony-forming units per milliliter $(\mathrm{CFU} / \mathrm{mL})$. This initial solution was diluted 1:100 to achieve a final concentration of $2 * 10^{6} \mathrm{CFU} / \mathrm{mL}$, and the inoculum was stored at room temperature until challenging the birds. Parallel to inoculation, the total bacterial count was determined to confirm the actual concentration of the inoculum. Due to technical problems with determining the optical density, animals were inoculated with $2 * 10^{4}$ $\mathrm{CFU} / \mathrm{mL}$ at the day of placement and again with a second inoculum containing $2 * 10^{8} \mathrm{CFU} / \mathrm{mL}$ at day three. Antimicrobial susceptibility testing of our strain was performed prior to the trial. EC14 was sensitive to lincomycin and spectinomycin using the disk diffusion test (Oxoid GmbH).

\section{Qualitative microbiology via culture}

EC-isolation was performed on Columbia colistinnalidixic acid (CNA) agar (Oxoid $\mathrm{GmbH}$ ) from Amies medium swabs taken at necropsy. The plates were incubated for $24 \mathrm{~h}$ at $37{ }^{\circ} \mathrm{C}$ and then screened for colonies of EC (small, gray, mucoid colonies with slight alphahemolysis). Pure subcultures from respective colonies were produced on Columbia sheep blood agar. After another $24 \mathrm{~h}$ of incubation, catalase and oxidase testing were performed and Gram staining of colony material complemented the microbiological examination. Besides typical colony morphology, isolates were identified as EC when they were oxidase and catalase negative, and gram positive to gram variable ovoid cocci were seen under the light microscope. Bacterial isolates that were not reliably identified by these methods were further analyzed via $16 \mathrm{~S}$ rRNA partial gene sequencing at Microsynth AG, Lindau, Germany [52-54]. 
DNA isolation and quantitative real-time PCR

DNA isolation was performed from dry swabs using a commercial isolation kit (InnuPrep DNA Mini Kit, Analytik Jena AG, Jena, Germany) in accordance with the manufacturer's instructions with minor modification. Only $30 \mu \mathrm{L}$ of the elution buffer were used for the last step of the procedure instead of the recommended $200 \mu \mathrm{L}$. After determining the total DNA amount using the NanoDrop ${ }^{\circledR}$ ND-1000 Spectrophotometer (Thermo Fisher Scientific Inc., Wilmington, NC, USA), DNA was stored at $-20^{\circ} \mathrm{C}$ until further use.

Each sample was analyzed in duplicate using a modified set-up of a recently published real-time PCR assay [55]. Each run was performed on 96-well-plates (Applied Biosystems $^{\mathrm{TM}}$, Fisher Scientific GmbH, Schwerte, Germany) using the QuantStudio 3 Real-Time-PCR-System (Thermo Fisher Scientific Inc.). The same primers, probes, and polymerase were used as described in the respective article [55], but only half of the volume of all ingredients was used per well. Initial denaturation was set at $95^{\circ} \mathrm{C}$ for $10 \mathrm{~min}$, followed by 40 amplification cycles at $95^{\circ} \mathrm{C}$ for $15 \mathrm{~s}$, and $60^{\circ} \mathrm{C}$ for $60 \mathrm{~s}$. Mean Ct values above 36 were considered negative during data analysis.

\section{Characterization of cecal microbiota}

Sequencing of the V3-V4 hypervariable region of $16 \mathrm{~S}$ rRNA genes from nine cecal samples per group and day was performed as previously described [56]. Sequencing results were analyzed and classified with RDP Seqmatch using the Qiime software [57]. The OTU (operational taxonomic unit) discrimination level was set to $97 \%$. As we were interested in the enterococcal species found in the analysis of the cecal microbiota, we tried to identify the most abundant OTUs that were assigned to the genus Enterococcus by using the NCBI Basic Local Alignment Search Tool (BLAST).

\section{Statistical analysis}

Data were statistically analyzed with SAS Enterprise Guide (Version 7.15, SAS Institute Inc., Cary, NC, USA) and graphs were created using GraphPad Prism (Version 9.2, GraphPad Software, LLC, San Diego, CA, USA). A descriptive statistical analysis was performed for clinical signs, pathology, and the composition of cecal microbiota at different taxonomic levels. Results from bacteriological examination via culture and real-time PCR were compared between groups using the Fisher's Exact Test. Results from culture and real-time PCR within the same group were compared using the Kappa coefficient [58] and McNemar's Test. Differences were considered significant at $p \leq 0.05$. In order to determine within sample diversity (alpha diversity), the diversity estimators Observed species, Chao1 index, and Shannon index were calculated in Qiime [57]. Differences in alpha diversity between groups were analyzed using the Kruskal-Wallis test and Mann-Whitney $U$ test, as conditions of normality and heterogeneity of variance were not met. The Bonferroni-Holm correction method for multiple testing was used to adjust $p$-values where applicable [59]. Principal coordinate analysis (PCoA) based on the weighted UniFrac analysis implemented in Qiime was used to visualize beta diversity.

\section{Supplementary Information}

The online version contains supplementary material available at https://doi. org/10.1186/s13099-021-00467-9.

Additional file 1. Beta diversity of cecal microbiota visualized in principle coordinate analysis based on weighted UniFrac distance metric implemented in Qiime. Dots of the same color represent nine samples from one of the four study groups at one of the sampling days. NN non-infected, non-treated, NL non-infected, treated with lincomycinspectinomycin, EN EC-infected, non-treated, EL EC-infected, treated with lincomycin-spectinomycin

Acknowledgements

The authors would like to thank Ilka Baumann, Susanne Nagel, Melanie Bode, and Sonja Bernhardt for their excellent technical support.

\section{Authors' contributions}

AJ, JS, and SR planned and designed the experiments. JS conducted the animal experiments, performed laboratory work and statistical analysis, and drafted the first manuscript. DK and MC performed the sequencing of cecal microbiota. AJ, IR, and SR reviewed and edited the manuscript. All authors read and approved the final manuscript.

\section{Funding}

Open Access funding enabled and organized by Projekt DEAL. This study was supported by the QS Fachgesellschaft Geflügel GmbH. IR, DK, and MC were partially supported by the project CZ.02.1.01/0.0/0.0/16_025/0007404 of the Ministry of Education, Youth and Sports of the Czech Republic. The publication was supported by Deutsche Forschungsgemeinschaft and University of Veterinary Medicine Hannover, Foundation within the funding programme Open Access Publishing

Availability of data and materials

All data generated or analyzed during this study are included in this published article and its supplementary information files.

\section{Declarations}

Ethics approval and consent to participate

The study design was approved by the Animal Ethics Committee of the University of Veterinary Medicine, Hannover and conducted in line with regulations on animal welfare and animal experimentation in Germany (33.19-42502-04-19/3170).

\section{Consent for publication}

Not applicable.

\section{Competing interests}

The authors declare that they have no competing interests.

\section{Author details}

${ }^{1}$ Clinic for Poultry, University of Veterinary Medicine Hannover, Buenteweg 17, 30559 Hannover, Germany. ${ }^{2}$ Veterinary Research Institute, Hudcova 296/70, 62100 Brno, Czech Republic. 
Received: 14 September 2021 Accepted: 22 November 2021

Published online:

\section{References}

1. Talebi A, Taifebagherlu J, Sharifi A. Delkhosh-Kasmaie F Spondylitis in broiler breeder farms in West-Azerbaijan province, Iran: clinical report. Vet Res Forum. 2016;7(4):353-5.

2. Jung A, Rautenschlein S (2014) Comprehensive report of an Enterococcus cecorum infection in a broiler flock in Northern Germany. BMC Vet Res 10:311. https://doi.org/10.1186/s12917-014-0311-7

3. Aitchison H, Poolman P, Coetzer M, Griffiths C, Jacobs J, Meyer M, Bisschop S (2014) Enterococcal-related vertebral osteoarthritis in South African broiler breeders: A case report. J S Afr Vet Assoc 85 (1):1077. https://doi.org/10.4102/jsava.v85i1.1077

4. Robbins KM, Suyemoto MM, Lyman RL, Martin MP, Barnes HJ, Borst LB (2012) An outbreak and source investigation of enterococcal spondylitis in broilers caused by Enterococcus cecorum. Avian Dis 56 (4):768-773. https://doi.org/10.1637/10253-052412-Case.1

5. Makrai L, Nemes C, Simon A, Ivanics E, Dudas Z, Fodor L, Glavits R (2011) Association of Enterococcus cecorum with vertebral osteomyelitis and spondylolisthesis in broiler parent chicks. Acta Vet Hung 59 (1):11-21. https://doi.org/10.1556/AVet.59.2011.1.2

6. Stalker MJ, Brash ML, Weisz A, Ouckama RM, Slavic D (2010) Arthritis and osteomyelitis associated with Enterococcus cecorum infection in broiler and broiler breeder chickens in Ontario, Canada. J Vet Diagn Invest 22 (4):643-645. https://doi.org/10.1177/104063871002200426

7. Herdt Pd, Defoort P, Steelant Jv, Swam H, Tanghe L, Goethem Sv, Vanrobaeys MJVDT (2009) Enterococcus cecorum osteomyelitis and arthritis in broiler chickens. 78 (1):44-48

8. Devriese LA, Cauwerts K, Hermans K, Wood AM (2002) Enterococcus cecorum septicemia as a cause of bone and joint lesions resulting in lameness in broiler chickens. Vlaams Diergeneeskundig Tijdschrift 71 (3):219-221

9. Borst LB, Suyemoto MM, Sarsour AH, Harris MC, Martin MP, Strickland JD Oviedo EO, Barnes HJ (2017) Pathogenesis of enterococcal spondylitis caused by Enterococcus cecorum in broiler chickens. Vet Pathol 54 (1):61-73. https://doi.org/10.1177/0300985816658098

10. Kense MJ, Landman WJ (2011) Enterococcus cecorum infections in broiler breeders and their offspring: molecular epidemiology. Avian Pathol 40 (6):603-612. https://doi.org/10.1080/03079457.2011.619165

11. Martin LT, Martin MP, Barnes HJ (2011) Experimental reproduction of enterococcal spondylitis in male broiler breeder chickens. Avian Dis 55 (2):273-278. https://doi.org/10.1637/9614-121410-Reg.1

12. Devriese LA, Ceyssens K, Haesebrouck F. Characteristics of Enterococcus cecorum strains from the intestines of different animal species. Lett Appl Microbiol. 1991;12(4):137-9. https://doi.org/10.1111/j.1472-765X.1991. tb00524.x.

13. Devriese LA, Hommez J, Wijfels R, Haesebrouck F (1991) Composition of the enterococcal and streptococcal intestinal flora of poultry. J Appl Bacteriol 71 (1):46-50

14. Borst LB, McLamb KA, Suyemoto MM, Chen LR, Levy MG, Sarsour AH, Cordova HA, Barnes HJ, Oviedo-Rondon EO. Coinfection with Eimeria spp. decreases bacteremia and spinal lesions caused by pathogenic Enterococcus cecorum. Anim Feed Sci Technol. 2019;250:59-68. https://doi.org/ 10.1016/j.anifeedsci.2018.09.014

15. Dolka B, Chrobak-Chmiel D, Makrai L, Szeleszczuk P (2016) Phenotypic and genotypic characterization of Enterococcus cecorum strains associated with infections in poultry. BMC Vet Res 12 (1):129. https://doi.org/10. 1186/s12917-016-0761-1

16. Jackson CR, Kariyawasam S, Borst LB, Frye JG, Barrett JB, Hiott LM, Woodley TA (2015) Antimicrobial resistance, virulence determinants and genetic profiles of clinical and nonclinical Enterococcus cecorum from poultry. Lett Appl Microbiol 60 (2):111-119. https://doi.org/10.1111/lam. 12374

17. Jung A, Metzner M, Ryll M (2017) Comparison of pathogenic and nonpathogenic Enterococcus cecorum strains from different animal species. BMC Microbiol 17 (1):33. https://doi.org/10.1186/s12866-017-0949-y
18. Logue CM, Andreasen CB, Borst LB, Eriksson H, Hampson DJ, Sanchez S, Fulton RM (2020) Other Bacterial Diseases. In: Diseases of Poultry. pp. 9951085. https://doi.org/10.1002/9781119371199.ch23

19. Kristich CJ, Rice LB, Arias CA. Enterococcal infection-treatment and antibiotic resistance. In: Gilmore MS, Clewell DB, Ike Y, et al., editors. Enterococci: from commensals to leading causes of drug resistant infection. U.S. National Institutes of Health NCBI Bookshelf; 2014.

20. Borst LB, Suyemoto MM, Robbins KM, Lyman RL, Martin MP, Barnes HJ (2012) Molecular epidemiology of Enterococcus cecorum isolates recovered from enterococcal spondylitis outbreaks in the southeastern United States. Avian Pathol 41 (5):479-485. https://doi.org/10.1080/03079457. 2012.718070

21. Suyemoto MM, Barnes HJ, Borst LB (2017) Culture methods impact recovery of antibiotic-resistant Enterococci including Enterococcus cecorum from pre- and postharvest chicken. Lett Appl Microbiol 64 (3):210-216. https://doi.org/10.1111/lam.12705

22. Sharma P, Gupta SK, Barrett JB, Hiott LM, Woodley TA, Kariyawasam S, Frye JG, Jackson CR (2020) Comparison of Antimicrobial Resistance and PanGenome of Clinical and Non-Clinical Enterococcus cecorum from Poultry Using Whole-Genome Sequencing. Foods 9 (6):686. https://doi.org/10. 3390/foods 9060686

23. Veterinary medicinal products containing a combination of lincomycin and spectinomycin to be administered orally to pigs and, or poultry. https://www.ema.europa.eu/en/medicines/veterinary/referrals/veter inary-medicinal-products-containing-combination-lincomycin-spect inomycin-be-administered-orally. Accessed 25 Feb 2021

24. Wei S, Morrison M, Yu Z. Bacterial census of poultry intestinal microbiome. Poult Sci. 2013;92(3):671-83. https://doi.org/10.3382/ps.2012-02822.

25. Mancabelli L, Ferrario C, Milani C, Mangifesta M, Turroni F, Duranti S, Lugli GA, Viappiani A, Ossiprandi MC, van Sinderen D, Ventura M (2016) Insights into the biodiversity of the gut microbiota of broiler chickens. Environ Microbiol 18 (12):4727-4738. https://doi.org/10.1111/1462-2920.13363

26. Torok VA, Hughes RJ, Mikkelsen LL, Perez-Maldonado R, Balding K, MacAlpine R, Percy NJ, Ophel-Keller K (2011) Identification and characterization of potential performance-related gut microbiotas in broiler chickens across various feeding trials. Appl Environ Microbiol 77 (17):5868-5878. https://doi.org/10.1128/Aem.00165-11

27. Torok VA, Hughes RJ, Ophel-Keller K, Ali M, Macalpine R (2009) Influence of different litter materials on cecal microbiota colonization in broiler chickens. Poult Sci 88 (12):2474-2481. https://doi.org/10.3382/ps. 2008-00381

28. Torok VA, Allison GE, Percy NJ, Ophel-Keller K, Hughes RJ (2011) Influence of antimicrobial feed additives on broiler commensal posthatch gut microbiota development and performance. Appl Environ Microbiol 77 (10):3380-3390. https://doi.org/10.1128/AEM.02300-10

29. Oakley BB, Vasconcelos EJR, Diniz P, Calloway KN, Richardson E, Meinersmann RJ, Cox NA, Berrang ME (2018) The cecal microbiome of commercial broiler chickens varies significantly by season. Poult Sci 97 (10):3635-3644. https://doi.org/10.3382/ps/pey214

30. Torok VA, Ophel-Keller K, Loo M, Hughes RJ (2008) Application of methods for identifying broiler chicken gut bacterial species linked with increased energy metabolism. Appl Environ Microbiol 74 (3):783-791. https://doi.org/10.1128/AEM.01384-07

31. Stanley D, Geier MS, Hughes RJ, Denman SE, Moore RJ (2013) Highly variable microbiota development in the chicken gastrointestinal tract. PLoS One 8 (12):e84290. https://doi.org/10.1371/journal.pone.0084290

32. Yin Y, Lei F, Zhu L, Li S, Wu Z, Zhang R, Gao GF, Zhu B, Wang X (2010) Exposure of different bacterial inocula to newborn chicken affects gut microbiota development and ileum gene expression. ISME J 4 (3):367-376. https://doi.org/10.1038/ismej.2009.128

33. Debnam AL, Jackson CR, Avellaneda GE, Barrett JB, Hofacre CL (2005) Effect of growth promotant usage on enterococci species on a poultry farm. Avian Dis 49 (3):361-365. https://doi.org/10.1637/7328-011405R.1

34. Grund A, Rautenschlein S, Jung A (2021) Tenacity of Enterococcus cecorum at different environmental conditions. J Appl Microbiol 130 (5):1494-1507. https://doi.org/10.1111/jam.14899

35. Remiot $P$, Panaget $G$, Chataigner $E$, Chevalier D. Enterococcus cecorum in broilers: a survey in farm to identify risky zootechnical practices. 13èmes Journées de la Recherche Avicole et Palmipèdes à Foie Gras, Tours, France, 20 et 21 mars 2019. 2019:116-20. 
36. Stepien-Pysniak D, Marek A, Banach T, Adaszek L, Pyzik E, Wilczynski J, Winiarczyk S (2016) Prevalence and antibiotic resistance of Enterococcus strains isolated from poultry. Acta Vet Hung 64 (2):148-163. https://doi. org/10.1556/004.2016.016

37. Skraban J, Dzeroski S, Zenko B, Tusar L, Rupnik M (2013) Changes of poultry faecal microbiota associated with Clostridium difficile colonisation. Vet Microbiol 165 (3-4):416-424. https://doi.org/10.1016/j.vetmic.2013.04. 014

38. Juricova H, Videnska P, Lukac M, Faldynova M, Babak V, Havlickova H, Sisak F, Rychlik I (2013) Influence of Salmonella enterica serovar enteritidis infection on the development of the cecum microbiota in newly hatched chicks. Appl Environ Microbiol 79 (2):745-747. https://doi.org/10.1128/ AEM.02628-12

39. Rychlik I. Composition and function of chicken gut microbiota. Animals. 2020;10(1):103.

40. Lu J, Idris U, Harmon B, Hofacre C, Maurer JJ, Lee MD. Diversity and succession of the intestinal bacterial community of the maturing broiler chicken. Appl Environ Microbiol. 2003;69(11):6816-24.

41. Nolan LK, Vaillancourt JP, Barbieri NL, Logue CM (2020) Colibacillosis. In: Diseases of Poultry. pp. 770-830. https://doi.org/10.1002/9781119371199. ch18

42. Callaway TR, Edrington TS, Anderson RC, Harvey RB, Genovese KJ, Kennedy CN, Venn DW, Nisbet DJ (2008) Probiotics, prebiotics and competitive exclusion for prophylaxis against bacterial disease. Anim Health Res Rev 9 (2):217-225. https://doi.org/10.1017/S1466252308001540

43. Goren E, de Jong WA, Doornenbal P (1988) Therapeutic efficacy of medicating drinking water with spectinomycin and lincomycin-spectinomycin in experimental Escherichia coli infection in poultry. Vet Q 10 (3):191-197. https://doi.org/10.1080/01652176.1988.9694170

44. Ahmed AM, Shimamoto T, Shimamoto T (2013) Molecular characterization of multidrug-resistant avian pathogenic Escherichia coli isolated from septicemic broilers. Int J Med Microbiol 303 (8):475-483. https://doi. org/10.1016/j.ijmm.2013.06.009

45. Osman KM, Kappell AD, Elhadidy M, ElMougy F, El-Ghany WAA, Orabi A, Mubarak AS, Dawoud TM, Hemeg HA, Moussa IMI, Hessain AM, Yousef HMY (2018) Poultry hatcheries as potential reservoirs for antimicrobialresistant Escherichia coli: A risk to public health and food safety. Sci Rep 8 (1):5859. https://doi.org/10.1038/s41598-018-23962-7

46. Pandey KR, Naik SR, Vakil BV (2015) Probiotics, prebiotics and synbiotics-a review. J Food Sci Technol 52 (12):7577-7587. https://doi.org/10.1007/ s13197-015-1921-1

47. Dec M, Nowaczek A, Urban-Chmiel R, Stepien-Pysniak D, Wernicki A (2018) Probiotic potential of Lactobacillus isolates of chicken origin with anti-Campylobacter activity. J Vet Med Sci 80 (8):1195-1203. https://doi. org/10.1292/jvms.18-0092

48. Tabashsum Z, Peng M, Alvarado-Martinez Z, Aditya A, Bhatti J, Romo PB, Young A, Biswas D (2020) Competitive reduction of poultry-borne enteric bacterial pathogens in chicken gut with bioactive Lactobacillus casei. Sci Rep 10 (1):16259. https://doi.org/10.1038/s41598-020-73316-5

49. Wang S, Peng Q, Jia HM, Zeng XF, Zhu JL, Hou CL, Liu XT, Yang FJ, Qiao SY (2017) Prevention of Escherichia coli infection in broiler chickens with Lactobacillus plantarum B1. Poult Sci 96 (8):2576-2586. https://doi.org/ 10.3382/ps/pex061

50. Jung A, Chen LR, Suyemoto MM, Barnes HJ, Borst LB (2018) A Review of Enterococcus cecorum Infection in Poultry. Avian Dis 62 (3):261-271. https://doi.org/10.1637/11825-030618-Review.1

51. Aviagen (2018) Ross 308 Broiler Management Handbook. https://en.aviag en.com/brands/ross/products/ross-308. Accessed 18 Aug 2021

52. Mignard S, Flandrois JP (2006) $16 \mathrm{~S}$ rRNA sequencing in routine bacterial identification: a 30-month experiment. J Microbiol Methods 67 (3):574-581. https://doi.org/10.1016/j.mimet.2006.05.009

53. Patel JB (2001) 165 rRNA gene sequencing for bacterial pathogen identification in the clinical laboratory. Mol Diagn 6 (4):313-321. https://doi.org/ 10.1054/modi.2001.29158

54. Wilson KH, Blitchington RB, Greene RC (1990) Amplification of bacterial 16S ribosomal DNA with polymerase chain reaction. J Clin Microbiol 28 (9):1942-1946. https://doi.org/10.1128/JCM.28.9.1942-1946.1990

55. Jung A, Petersen $H$, Teske L, Rautenschlein S. Colonization patterns of Enterococcus cecorum in two different broiler production cycles detected with a newly developed quantitative real-time PCR. BMC Microbiol. 2017;17(1):106. https://doi.org/10.1186/s12866-017-1021-7.
56. Kollarcikova M, Kubasova T, Karasova D, Crhanova M, Cejkova D, Sisak F, Rychlik I. Use of $16 \mathrm{~S}$ rRNA gene sequencing for prediction of new opportunistic pathogens in chicken ileal and cecal microbiota. Poult Sci. 2019;98(6):2347-53. https://doi.org/10.3382/ps/pey594.

57. Caporaso JG, Kuczynski J, Stombaugh J, Bittinger K, Bushman FD, Costello EK, Fierer N, Peña AG, Goodrich JK, Gordon Jl, Huttley GA, Kelley ST, Knights D, Koenig JE, Ley RE, Lozupone CA, McDonald D, Muegge BD, Pirrung M, Reeder J, Sevinsky JR, Turnbaugh PJ, Walters WA, Widmann J, Yatsunenko T, Zaneveld J, Knight R. QIIME allows analysis of high-throughput community sequencing data. Nat Methods. 2010;7(5):335-6. https://doi. org/10.1038/nmeth.f.303.

58. Landis JR, Koch GG. The measurement of observer agreement for categorical data. Biometrics. 1977;33(1):159-74. https://doi.org/10.2307/ 2529310.

59. Holm S. A simple sequentially rejective multiple test procedure. Scand J Stat. 1979;6(2):65-70.

\section{Publisher's Note}

Springer Nature remains neutral with regard to jurisdictional claims in published maps and institutional affiliations.

Ready to submit your research? Choose BMC and benefit from:

- fast, convenient online submission

- thorough peer review by experienced researchers in your field

- rapid publication on acceptance

- support for research data, including large and complex data types

- gold Open Access which fosters wider collaboration and increased citations

- maximum visibility for your research: over $100 \mathrm{M}$ website views per year

At BMC, research is always in progress.

Learn more biomedcentral.com/submissions 